\title{
Mitral Valve Surgery for Severe Mitral Regurgitation and Dilated Cardiomyopathy-A Bridge to Transplant: Case Report and a Review of Literature
}

\author{
Daisuke Kobayashi, MD, ${ }^{*}$ Michael D. Pettersen, MD, ${ }^{*}$ Henry L. Walters, III, MD, ${ }^{\dagger}$ and \\ Sanjeev Aggarwal, MD* \\ *Division of Cardiology, Carman and Ann Adams Department of Pediatrics and ${ }^{\dagger}$ Department of Cardiovascular Surgery, \\ Children's Hospital of Michigan, Wayne State University School of Medicine, Detroit, Mich, USA
}

\begin{abstract}
A B S T R A C T
We report a child with myocardial necrosis, dilated cardiomyopathy, and severe mitral valve (MV) regurgitation following neonatal enteroviral myocarditis. He underwent MV annuloplasty at 18 months and MV replacement at 3 years of age. He remains asymptomatic on medical therapy at 8 years of age. Mitral valve surgery may stabilize the evolution of dilated cardiomyopathy and delay the ultimate need for heart transplant.
\end{abstract}

Key Words. Mitral Valve Annuloplasty; Mitral Valve Replacement; Enteroviral Myocarditis; Myocardial Necrosis; Dilated Cardiomyopathy

\section{Introduction}

$\mathrm{N}_{\mathrm{in}}^{\mathrm{e}}$ eonatal enteroviral myocarditis is a devastating disease and often leads to severe cardiac consequences such as dilated cardiomyopathy (DCM), which, in turn, is associated with $31 \%$ reported mortality. ${ }^{1}$ In DCM, functional mitral regurgitation (MR) develops because of incomplete leaflet coaptation secondary to a change in the mitral valve (MV) apparatus and altered ventricular geometry. ${ }^{2}$ Mitral regurgitation may cause further annular dilation, progressive worsening of $\mathrm{MR}$, and left ventricular (LV) dysfunction. In a study of 42 children with idiopathic DCM, MR severity was an independent risk factor for worse clinical status and death or transplantation. ${ }^{3}$

Dilated cardiomyopathy is the most common indication for orthotopic heart transplant (OHT) in children. ${ }^{4}$ Although OHT is a definitive treatment, donor availability is limited, recipients require lifelong antirejection medications, and the life span of a transplanted heart is limited. Therefore, nontransplant surgical therapies of end-stage cardiomyopathy are topics of recent investigations and may enable OHT to be postponed or avoided, although experience in children is limited. We report a case with severe MR associated with DCM and myocardial necrosis following neonatal enteroviral myocarditis, in which MV annuloplasty and subsequent mitral prosthetic valve replacement successfully stabilized the evolution of DCM and has obviated need for OHT thus far. In addition, we present a review of the literature on outcomes of surgical approaches for DCM in children.

\section{Case Report}

A 9-day-old, full-term male infant presented with cardiogenic shock. He was born via normal vaginal delivery with a birth weight of $3600 \mathrm{~g}$ without significant antenatal or immediate neonatal complications. An echocardiogram at presentation showed normal intracardiac anatomy, normal aortic arch, severely dilated $\mathrm{LV}$, and severe $\mathrm{LV}$ dysfunction with severe mitral and tricuspid regurgitation. Because of paradoxical ventricular septal motion, the LV ejection fraction (LVEF) or shortening fraction could not be measured accurately. The estimated right ventricular systolic pressure 


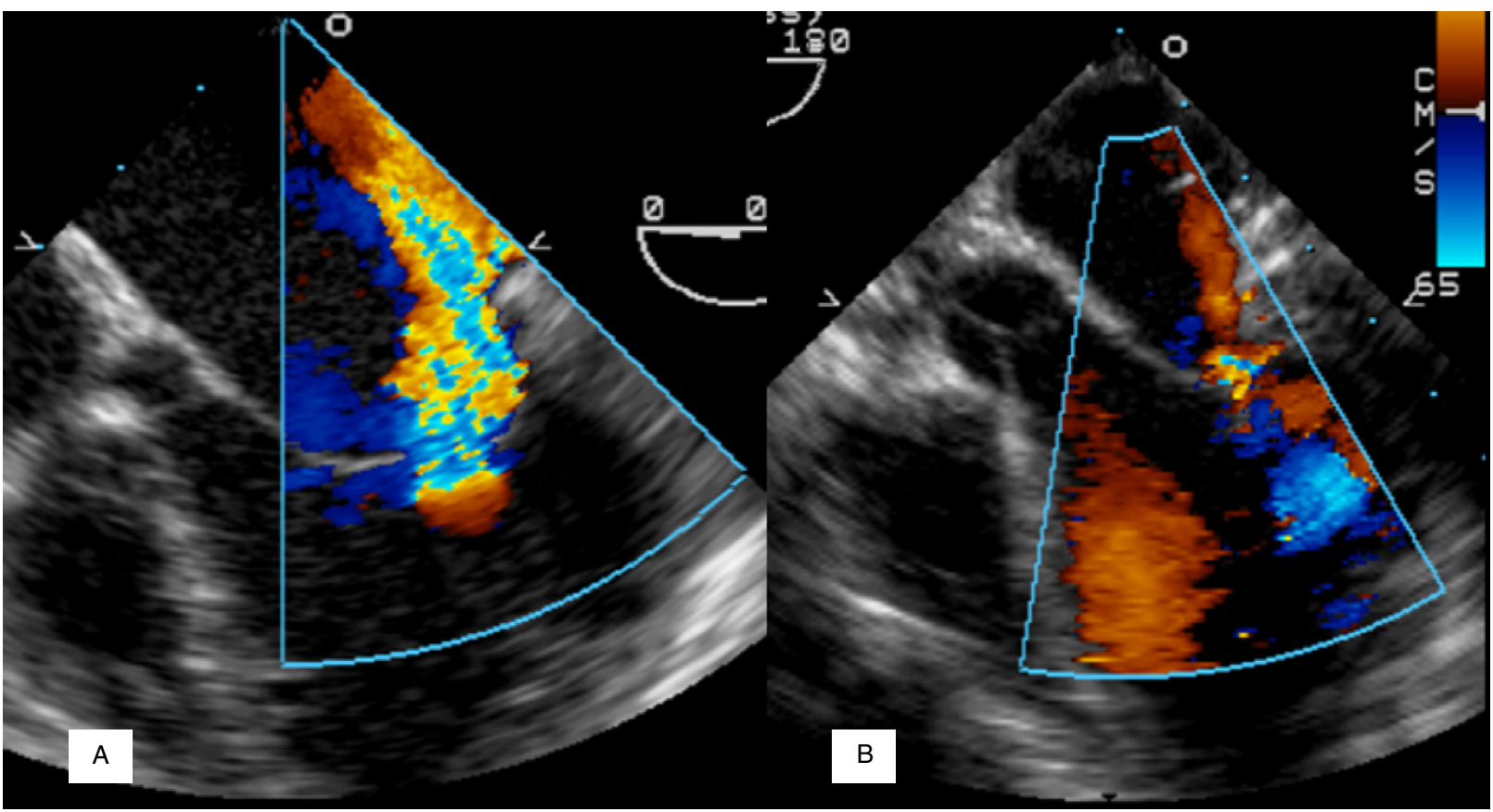

Figure 1. Intraoperative transesophageal echocardiography (A) before and (B) after mitral valve annuloplasty. Mitral annulus and mitral regurgitation was significantly reduced.

(RVSP) was $56 \mathrm{~mm} \mathrm{Hg}$ plus right atrial pressure. The coronary arteries appeared to have a normal origin. There was a small pericardial effusion. Troponin I was $>50 \mathrm{ng} / \mathrm{mL}$ (normal range $<2$ ) and CK-MB was $131 \mathrm{ng} / \mathrm{mL}$ (normal range $<10$ ). Rectal and nasopharyngeal viral culture and polymerase chain reaction in blood were found to be positive for enterovirus confirming a diagnosis of enteroviral myocarditis. The electrocardiogram (ECG) showed deep and wide Q wave in V6 and ST elevation in inferior leads and ST depression in the right precordial leads, suggestive of myocardial ischemia. The infant was supported hemodynamically by multiple inotropes, and enteroviral infection was treated with the antienteroviral agent pleconaril and intravenous immuoglobulin. He was discharged home 36 days later with improvement of LV function. The LV function (LVEF 44\%) was moderately decreased with trivial MR and akinesia of the LV posterior basilar region.

During follow-up, he was noted to have failed to thrive, and at 17 months of age, echocardiography showed severe $M R$ and $L V$ dilation (LV end-diastolic dimension [LVEDD] $43 \mathrm{~mm}$, $\mathrm{z}$-score +6.2) and an increase in the estimated RVSP. LVEF was $48 \%$. Cardiac catheterization was performed to assess the hemodynamics and showed a mildly elevated RVSP $(36 \mathrm{~mm} \mathrm{Hg})$. The left pulmonary capillary wedge pressure (mean $12 \mathrm{~mm} \mathrm{Hg}$ ) and LVED pressure $(11 \mathrm{~mm} \mathrm{Hg}$ ) were elevated with no evidence of significant MV stenosis. The calculated pulmonary vascular resistance (PVR) was 2.2 indexed Woods units. Coronary angiography showed no focal stenosis or aneurysm. Mitral valve annuloplasty was performed at 18 months of age as a bridge to OHT. Intraoperative inspection revealed a large, oval-shaped, bright akinetic area measuring $6 \times 4 \mathrm{~cm}$ in the postero-basal wall of the LV. The MV was morphologically normal with a dilated annulus measuring $22 \mathrm{~mm}$ in diameter. A horizontal mattress, pledgetted 4-0 Prolene (Ethicon, Somerville, NJ, USA) suture was placed from the inferior commissure along the posterior-inferior portion of the annulus. The MV annulus diameter was tightened to $16 \mathrm{~mm}$. Intraoperative transesophageal echocardiography showed trivial MR (Figure 1). However, as inotropes were weaned, by discharge, he developed moderate MR with LVEF of $50 \%$. He continued to be managed medically with captopril, digoxin, furosemide, and aspirin.

Mitral regurgitation and LV dysfunction gradually progressed over the next 1 year. At 32 months of age, echocardiography showed severe LV dilation (LVEDD $44 \mathrm{~mm}, z$-score 5.1) with severe MR and LVEF of $50 \%$. Except for failure to 

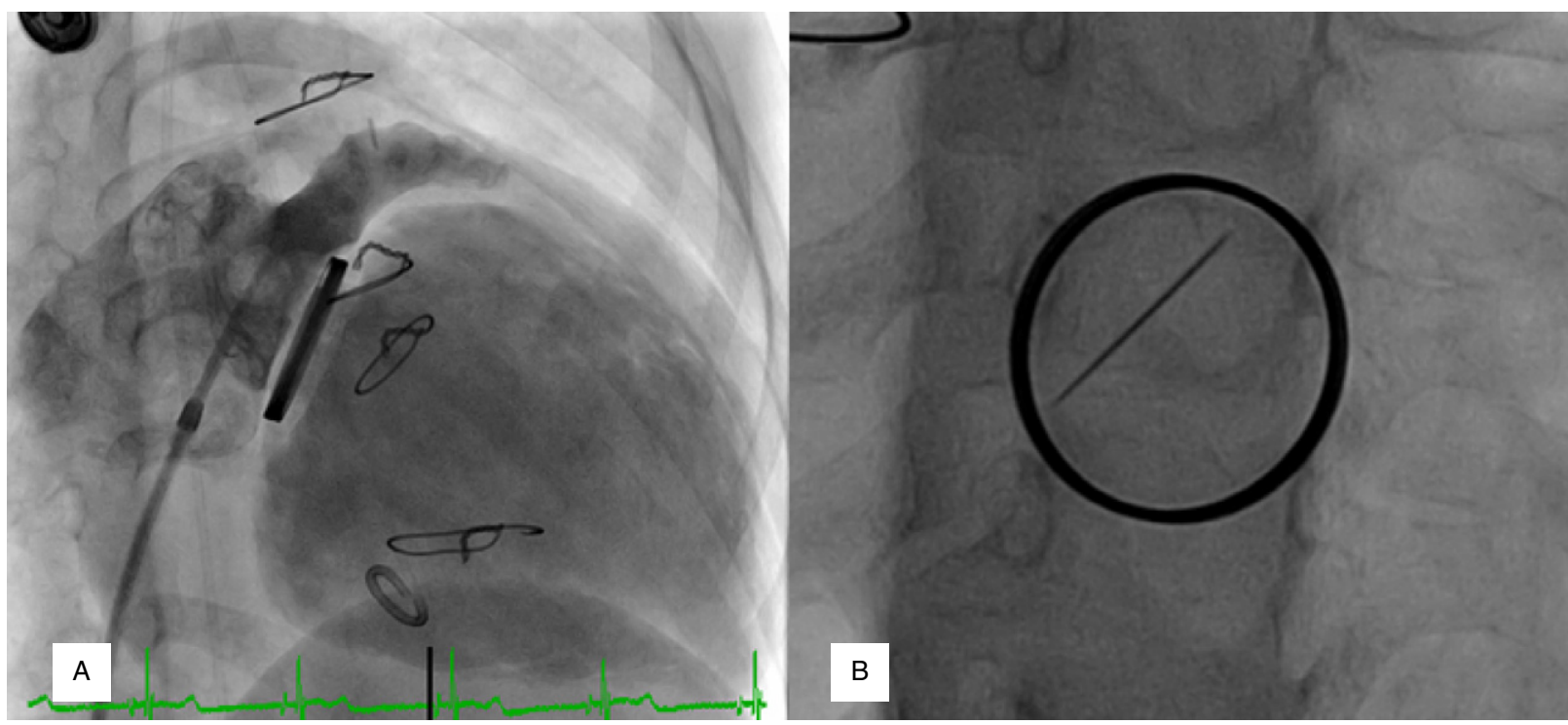

Figure 2. Cardiac catheterization at 7 years old, prior to surgical debridement of mitral prosthetic valve. (A) RAO $30^{\circ}$. Using the transseptal approach, the Berman angio catheter was advanced into the left atrium. Angiography in the left atrium showed dilated left atrium and left ventricle with prosthetic mitral valve. (B) Fluoroscopy of prosthetic mitral valve (21 mm diameter St. Jude) showed that posterior leaflet was immobile.

thrive, he remained asymptomatic and weighed $11.3 \mathrm{~kg}$ (second percentile). At 3 years of age, cardiac catheterization was performed, and the LV was found to be moderately dilated with further elevation of the LVED pressures to $14 \mathrm{~mm} \mathrm{Hg}$ and a normal RVSP $(32 \mathrm{~mm} \mathrm{Hg})$. The PVR was 4.5 indexed Woods units. After extensive discussion, because of worsening of the LVED pressure and failure to thrive, the decision was made to perform MV replacement (MVR). The MV was replaced with a St. Jude $21 \mathrm{~mm}$ diameter mechanical prosthesis (St. Jude, Minneapolis, MN, USA). The postoperative course was uncomplicated. Postoperatively, he continued to remain free of respiratory symptoms and experienced an improvement in body weight on a medical regimen of coumadin and enalapril.

He did develop a gradual increase in the gradient across the prosthetic MV without an increase of the estimated RVSP. At 7 years of age, the echocardiogram showed that the mean pressure gradient across the prosthetic MV was significantly increased at $15 \mathrm{~mm} \mathrm{Hg}$ with an estimated RVSP of $58 \mathrm{~mm} \mathrm{Hg}$. Cardiac catheterization demonstrated a severely elevated left atrial (LA) pressure with a mean of $18 \mathrm{~mm} \mathrm{Hg}$ and a $\mathrm{V}$ wave of $30 \mathrm{~mm} \mathrm{Hg}$. The A wave of the LA pressure was similar to the LVED pressure of $15 \mathrm{~mm} \mathrm{Hg}$. The RVSP was $59 \mathrm{~mm} \mathrm{Hg}$ (two-thirds of systemic). The PVR was 4.8 indexed Woods units. The pos- terior leaflet of the prosthetic MV was immobile on fluoroscopy (Figure 2). He underwent surgical debridement of the MV. Intraoperatively, significant psuedointima, fibrous ingrowth, and thrombus were noted around the entire MV annulus. After debridement, the valve regained normal function. At the latest follow-up at 8 years of age, he remains asymptomatic. Echocardiogram showed normal mobility of both leaflets of the prosthetic MV. The mean gradient across the MV was $5 \mathrm{~mm} \mathrm{Hg}$. The $\mathrm{LV}$ function was moderately reduced but unchanged with LVEF of $47 \%$. The estimated RVSP was $37 \mathrm{~mm} \mathrm{Hg}$ with a LVEDD of $51 \mathrm{~mm}(z$-score +5.2$)$.

\section{Discussion}

Mitral valve regurgitation is frequently associated with DCM and is reported to be associated with worsening of DCM and to be an independent risk factor for need for $\mathrm{OHT}^{3}$ An intervention to decrease MR leads to improvement in $L V$ volume and decreased LA volume overload and may prevent LV remodeling. Therefore, early repair of the MV has been tried in adults to obviate or delay the need for OHT. There is limited experience with early MV repair in children with DCM. In our case, first MV annuloplasty and subsequent MVR has averted the need for OHT until 8 years of age. 
Nontransplant surgical therapies of end-stage cardiomyopathy are rapidly evolving and include MVR, MV repair, and partial left ventriculectomy (PLV). We performed a literature review of nontransplant surgeries for DCM in children. ${ }^{2,5-17} \mathrm{~A}$ total of 33 children in the literature underwent MVR, MV repair, and/or PLV at a median age of 26 months (range 3 days-10.9 years) and a median weight of $7 \mathrm{~kg}$ (range 3.3-12 kg) (Table 1). Sixteen children underwent $M V$ repair, seven underwent PLV and MV repair, five underwent PLV, and five underwent MVR. Among those, two children had previous MV repair before MV repair, one had previous MV repair before MVR, and two had PLV before MVR. The follow-up duration of the reported cases is generally short. During the median follow-up of 7 months (range 1 week-17 years), the mortality rate was $15 \%$ at a median duration of 47 days (20 days-2 months) from the surgery. Eleven (33\%) children underwent OHT at a median duration of 7 months (range 1 month2.3 years) from the surgical intervention. The nontransplant surgical approaches acted as a bridge to OHT in these cases with fair efficacy (51\% survival without OHT). The severity of heart failure varies among patients with MR and DCM. Although successful MV surgery has been reported as an urgent rescue in patients with uncompensated heart failure, early postoperative mortality cannot be ignored. In the review of literature, most patients required presurgical intravenous inotropes but electively underwent nontransplant surgeries prior to the stage of uncompensated heart failure necessitating mechanical ventilator support. In contrast, two patients were on mechanical ventilation prior to the surgery: one patient was treated with extracorporeal membrane oxygenation and died 47 days after surgery, while the other patient was on high dose inotropes and mechanical ventilator underwent successful MV repair and subsequent OHT. ${ }^{14}$ For critically ill patients, ventricular assist device and/or extracorporeal cardiopulmonary membrane oxygenation may be safer choices of therapy as bridge to transplant. Nontransplant surgeries may have a role as urgent rescue and to wean inotropes and ventilation.

Mitral valve replacement is an alternative surgical option when MV repair is not possible for MV diseases. ${ }^{18}$ However, MVR is challenging in young children because of the limited availability of adequate-sized prosthesis, the need for anticoagulation, and the poor long-term outcome. ${ }^{18}$ In the multiinstitutional study of 139 children aged
$<5$ years undergoing MVR, the 10 -year survival was $74 \%$ with a 5 -year freedom from reoperation of $81 \% .{ }^{19}$ The risk of second MVR was highest in children who were $<2$ years old and received a prosthesis $<20 \mathrm{~mm}$ at the initial MVR. ${ }^{20}$ The reasons for a secondary MVR were prosthetic valve stenosis $83 \%$, thrombosis $14 \%$, and endocarditis $3 \% .{ }^{20}$ In our patient, MVR was performed using a $21 \mathrm{~mm}$ prosthetic valve at 3 years of age after a failed MV repair. Mitral valve replacement was selected at the second operation instead of repeated MV repair, because MVR was deemed to have a secure surgical outcome. Whereas, MV repair had a likelihood of failure again because of papillary muscle dysfunction.

The prosthetic valve was rescued by surgical debridement of fibrous tissue and thrombus, when prosthetic valve dysfunction occurred 4 years after insertion.

Neonatal enteroviral myocarditis is a devastating disease, which often leads to chronic heart failure, aneurysm formation within the $\mathrm{LV}$, and MR. ${ }^{1}$ In a review of 35 cases of neonatal enteroviral myocarditis, the mortality was $31 \%$ and $66 \%$ of survivors developed severe cardiac sequelae. ${ }^{1}$ Myocardial infarction has been reported in cases of neonatal enteroviral myocarditis. ${ }^{21}$ It is unclear whether enterovirus-induced myocardial necrosis mimics myocardial infarction. The difficulties in differentiating between neonatal myocardial infarction and myocarditis were discussed by de Vetten et al. ${ }^{22}$ Clinical presentation and diagnostic findings such as ECG changes and regional wall motion abnormalities are similar in both entities. Freund et al. advocated that the absence of the $\mathrm{R}$ wave and the presence of a new $\mathrm{Q}$ wave in I, II, and left precordial leads on ECG may be useful to diagnose neonatal myocarditis. ${ }^{1}$ In our case, myocardial infarction was suspected because of a Q wave on ECG and because of akinesia of the $\mathrm{LV}$ posterior basilar region on echocardiography. However, subsequent coronary angiography showed no discrete stenosis. Although the possibility of spontaneous recanalization of the coronary artery cannot be excluded, our case was likely to have an enterovirus-induced myocardial necrosis. In enterovirus-induced myocarditis in neonates, myocardial damage occurs by direct myocyte lysis and apoptotic damage to the infected myocytes. ${ }^{1}$ This myocyte necrosis may mimic a myocardial infarction in the absence of any localized ischemia. ${ }^{1}$ Subsequently necrosis is repaired by scar formation and aneurysm formation. Although the effect of enterovirus on the 


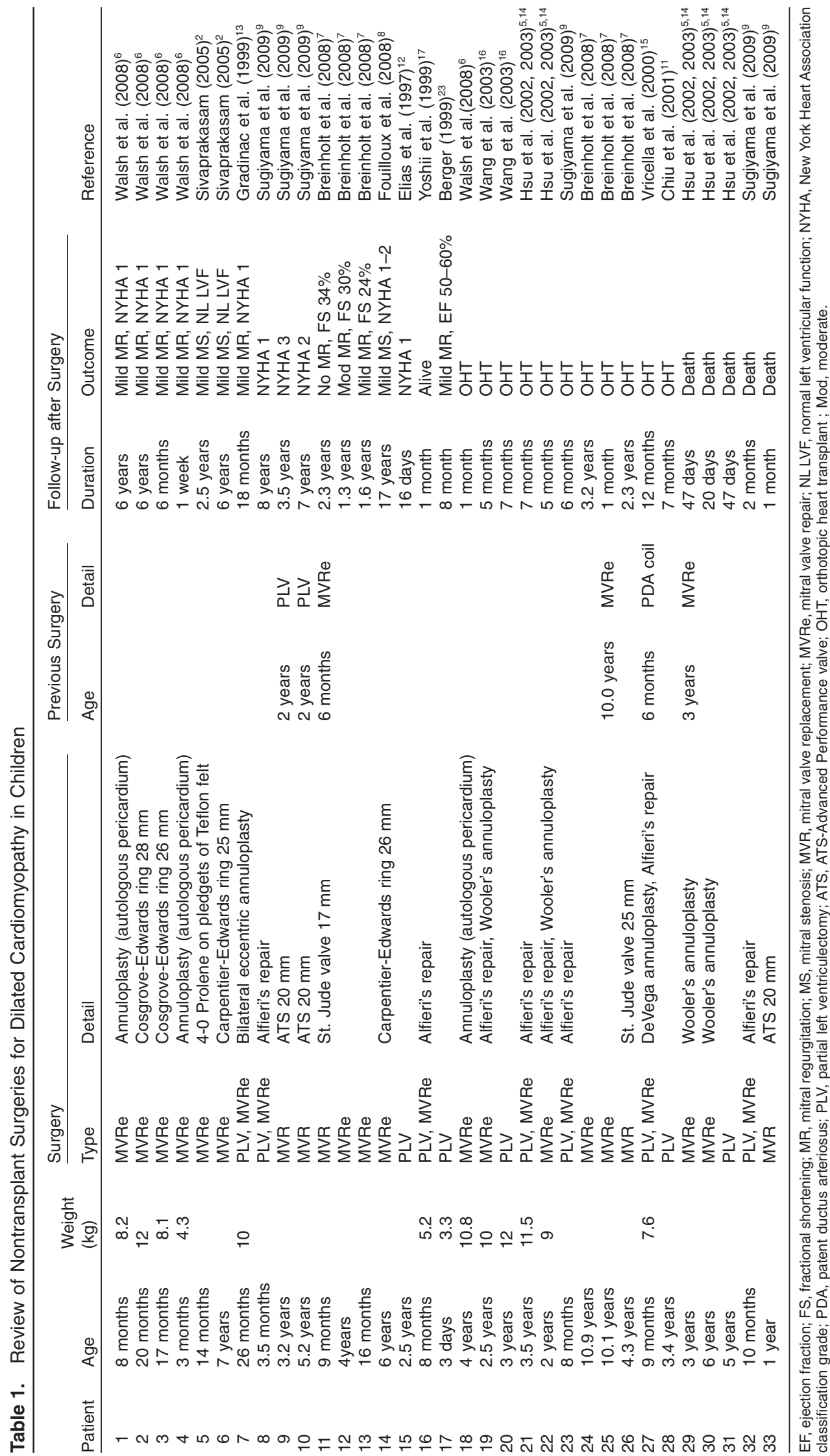


endothelium of the coronary vasculature is not well described, a microvascular coronary arteritis is a putative mechanism of entroviral myocarditisinduced infarction. ${ }^{21}$ Other postulated mechanisms included coronary thrombosis, coronary artery spasm, coronary embolism, and large vessel coronary arteritis. ${ }^{21}$ This could be a possible explanation for posterobasal aneursym formation in our case.

\section{Conclusion}

We report a challenging pediatric case with DCM and severe MR following neonatal enteroviral myocarditis in which MV surgery such as annuloplasty and prosthetic valve replacement successfully stabilized the evolution of DCM and has obviated the need for OHT thus far. In our review of the literature, we found a $51 \%$ rate of survival without OHT among 33 cases in which surgical interventions were performed. Mitral valve surgery may be considered as a therapeutic option in children with severe MR and DCM to obviate need for OHT.

\section{Author Contribution}

DK wrote the manuscript and collected the data for review of literature. MDP, HLW, and SA critically revised and approved it.

Corresponding Author: Daisuke Kobayashi, MD, Division of Cardiology, Carman and Ann Adams Department of Pediatrics, Children's Hospital of Michigan, 3901 Beaubien Boulevard, Detroit, MI 48201-2119, USA. Tel: (+1) 313-745-5481; Fax: $(+1)$ 313-993-0894; E-mail: dkobayas@dmc.org

Disclosure: No conflict of interest, grants, or other financial support.

Accepted in final form: December 8, 2011.

\section{References}

1 Freund MW, Kleinveld G, Krediet TG, van Loon AM, Verboon-Maciolek MA. Prognosis for neonates with enterovirus myocarditis. Arch Dis Child Fetal Neonatal Ed. 2010;95:F206-F212.

2 Sivaprakasam MC, Gnanapragasam JP, Salmon AP, et al. Annuloplasty of the regurgitant mitral valve after myocarditis in children. Ann Thorac Surg. 2005;80:1922-1924.

3 Fernandes FP, Manlhiot C, McCrindle BW, Mertens L, Kantor PF, Friedberg MK. Usefulness of mitral regurgitation as a marker of increased risk for death or cardiac transplantation in idiopathic dilated cardiomyopathy in children. Am 7 Cardiol. 2011;107:1517-1521.

4 Boucek MM, Aurora P, Edwards LB, et al. Registry of the International Society for Heart and Lung Transplantation: tenth official pediatric heart transplantation report-2007. 7 Heart Lung Transplant. 2007;26:796-807.

5 Hsu RB, Chien CY, Wang SS, Chu SH. Nontransplant cardiac surgery as a bridge to heart transplantation in pediatric dilated cardiomyopathy. Tex Heart Inst F. 2002;29:213-215.

6 Walsh MA, Benson LN, Dipchand AI, et al. Surgical repair of the mitral valve in children with dilated cardiomyopathy and mitral regurgitation. Ann Thorac Surg. 2008;85:2085-2088.

7 Breinholt JP, Fraser CD, Dreyer WJ, et al. The efficacy of mitral valve surgery in children with dilated cardiomyopathy and severe mitral regurgitation. Pediatr Cardiol. 2008;29:13-18.

8 Fouilloux V, Ghez O, Kreitmann B, Wernert F, Metras D. Mitral annuloplasty in dilated cardiomyopathy: eighteen-year follow up in a pediatric case. 7 Heart Valve Dis. 2008;17:639-641.

9 Sugiyama H, Hoshiai M, Naitoh A, Kadono T, Suzuki S, Sugita K. Outcome of non-transplant surgical strategy for end-stage dilated cardiomyopathy in young children. Circ 7. 2009;73:10451048.

10 Baba S, Doi H, Ikeda T, Komeda M, Nakahata T. A long-term follow-up of a girl with dilated cardiomyopathy after mitral valve replacement and septal anterior ventricular exclusion. 7 Cardiothorac Surg. 2009;4:53.

11 Chiu SN, Wu MH, Wang JK, et al. Heart transplantation and the Batista operation for children with refractory heart failure. Jpn Circ 7. 2001;65: 289-293.

12 Elias LM, Monteiro R, Fittaroni R, et al. [Partial ventriculectomy in a child-the Batista technique]. Arq Bras Cardiol. 1997;69:133-135.

13 Gradinac S, Jovanović I, Dukić M, et al. Partial left ventriculectomy in a two-year-old girl with dilated cardiomyopathy. 7 Heart Lung Transplant. 1999;18: 381-383.

14 Hsu RB, Chen RJ, Wu MH, Wang JK, Wang SS, Chu SH. Non-transplant cardiac surgery for endstage dilated cardiomyopathy in small children. 7 Heart Lung Transplant. 2003;22:94-97.

15 Vricella LA, Gundry SR, Larsen RL, Bailey LL. Successful myocardial volume reduction in a 9-month-old infant. Ann Thorac Surg. 2000;69: 1253-1255.

16 Wang SS, Hsu RB, Chen YS, et al. Heart transplantation and mitral valve repair in pediatric patients with refractory heart failure. Transplant Proc. 2003; $35: 463-465$. 
17 Yoshii S, Hosaka S, Takahashi W, et al. Partial left ventriculectomy in an infant with dilated cardiomyopathy. 7 Thorac Cardiovasc Surg. 1999;117:616-618.

18 Lee C, Lee CH, Kwak JG, et al. Long-term results after mitral valve repair in children. Eur 7 Cardiothorac Surg. 2010;37:267-272.

19 Caldarone CA, Raghuveer G, Hills CB, et al. Longterm survival after mitral valve replacement in children aged $<5$ years: a multi-institutional study. Circulation. 2001;104:I143-I147.

20 Raghuveer G, Caldarone CA, Hills CB, Atkins DL, Belmont JM, Moller JH. Predictors of prosthesis survival, growth, and functional status following mechanical mitral valve replacement in children aged $<5$ years, a multi-institutional study. Circulation. 2003;108:II174-II179.

21 Hornung TS, Bernard EJ, Howman-Giles RB, Sholler GF. Myocardial infarction complicating neonatal enterovirus myocarditis. 7 Paediatr Child Health. 1999;35:309-312.

22 de Vetten L, Bergman KA, Elzenga NJ, van Melle JP, Timmer A, Bartelds B. Neonatal myocardial infarction or myocarditis? Pediatr Cardiol. 2011;32: 492-497.

23 Berger S, Tweddell JS, Frommelt PC, Weinhaus L. Partial left ventriculectomy for dilated cardiomyopathy in a newborn. 7 Thorac Cardiovasc Surg. 1999; 117:1017-1018. 